\title{
A computational study of droplet-based bioprinting: Effects of viscoelasticity
}

Cite as: Phys. Fluids 31, 081901 (2019); https://doi.org/10.1063/1.5108824

Submitted: 02 May 2019 . Accepted: 25 July 2019 . Published Online: 16 August 2019

M. Nooranidoost (D), D. Izbassarov (D), S. Tasoglu (D), and M. Muradoglu (D)
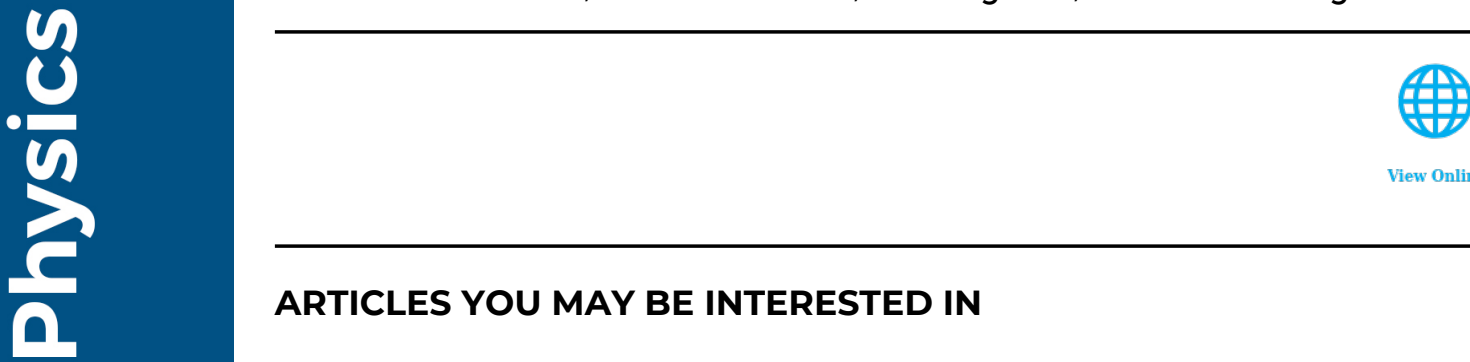

\section{ARTICLES YOU MAY BE INTERESTED IN}

Cell trapping in Y-junction microchannels: A numerical study of the bifurcation angle effect in inertial microfluidics

Physics of Fluids 31, 082003 (2019); https://doi.org/10.1063/1.5113516

Analytical prediction of electrowetting-induced jumping motion for droplets on hydrophobic substrates

Physics of Fluids 31, 081703 (2019); https://doi.org/10.1063/1.5109164

The role of surface wettability on the heat transfer in liquid-liquid two-phase flow in a microtube

Physics of Fluids 31, 082004 (2019); https://doi.org/10.1063/1.5093095

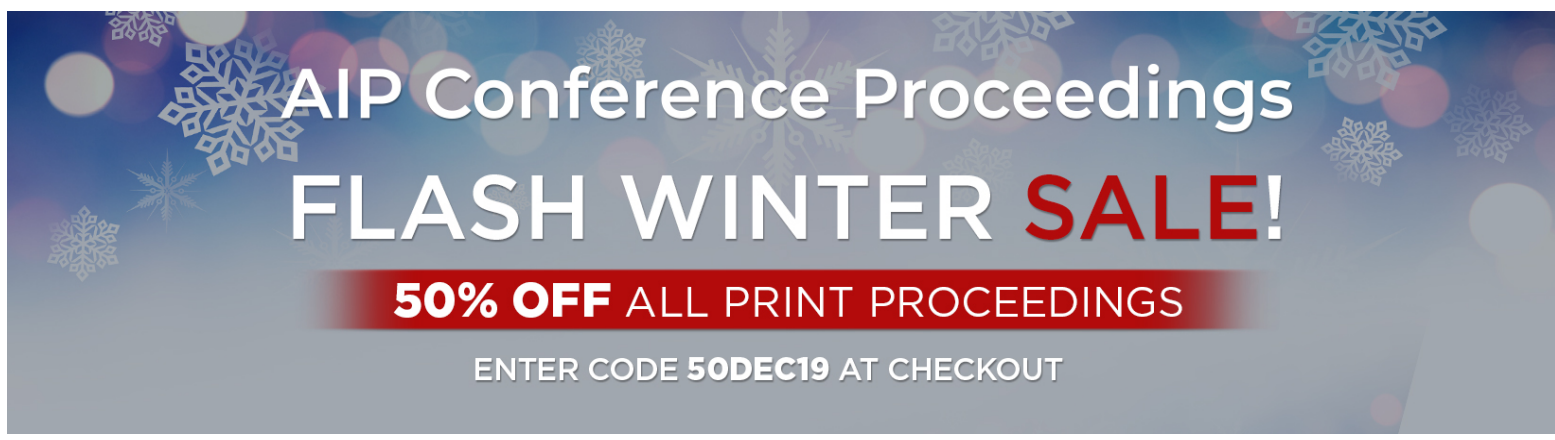




\title{
A computational study of droplet-based bioprinting: Effects of viscoelasticity
}

\author{
Cite as: Phys. Fluids 31, 081901 (2019); doi: 10.1063/1.5108824 \\ Submitted: 2 May 2019 - Accepted: 25 July 2019 • \\ Published Online: 16 August 2019
}

\author{
M. Nooranidoost, ${ }^{1, a)}$ (D) D. Izbassarov, ${ }^{1, b)}$ (D) S. Tasoglu, ${ }^{2}$ (D) and M. Muradoglu'
}

\begin{abstract}
AFFILIATIONS
${ }^{1}$ Department of Mechanical Engineering, Koc University, Rumelifeneri Yolu, 34450 Sariyer, Istanbul, Turkey

${ }^{2}$ Department of Mechanical Engineering, Department of Biomedical Engineering, Institute of Materials Science, Institute for Collaboration on Health, Intervention, and Policy, The Connecticut Institute for the Brain and Cognitive Sciences, University of Connecticut, Storrs, Connecticut 06269, USA
\end{abstract}

\author{
a) Present address: Department of Mechanical and Aerospace Engineering, University of Central Florida, Orlando, \\ Florida 32816, USA. \\ b) Present address: Linné Flow Center and SeRC, KTH Mechanics, SE 10044 Stockholm, Sweden. \\ c) Electronic mail: mmuradoglu@ku.edu.tr.
}

\begin{abstract}
Despite significant progress, cell viability continues to be a central issue in droplet-based bioprinting applications. Common bioinks exhibit viscoelastic behavior owing to the presence of long-chain molecules in their mixture. We computationally study effects of viscoelasticity of bioinks on cell viability during deposition of cell-loaded droplets on a substrate using a compound droplet model. The inner droplet, which represents the cell, and the encapsulating droplet are modeled as viscoelastic liquids with different material properties, while the ambient fluid is Newtonian. The model proposed by Takamatsu and Rubinsky ["Viability of deformed cells," Cryobiology 39(3), 243-251 (1999)] is used to relate cell deformation to cell viability. We demonstrate that adding viscoelasticity to the encapsulating droplet fluid can significantly enhance the cell viability, suggesting that viscoelastic properties of bioinks can be tailored to achieve high cell viability in droplet-based bioprinting systems. The effects of the cell viscoelasticity are also examined, and it is shown that the Newtonian cell models may significantly overpredict the cell viability.
\end{abstract}

Published under license by AIP Publishing. https://doi.org/10.1063/1.5108824

\section{INTRODUCTION}

Bioprinting is defined as the application of printing technology to deposit living cells onto a substrate. ${ }^{1-6}$ Recent developments in additive manufacturing have enabled printing of biocompatible materials, biochemicals, and living cells with precise spatial control over the placement of functional components on a substrate. ${ }^{1,3,5}$ Among the goals are to print tissues and organs suitable for transplantation and to create physiologically relevant disease ${ }^{7,8}$ and tissue models. ${ }^{9-11}$ However, bioprinting is still at an early stage in its development, and there is a long way from printing functional organs. Thus, much research is currently focused on the postprinting cell viability and functionality. ${ }^{12}$ In this respect, computational tools can be harnessed to understand the underlying factors affecting the cell viability and shape fidelity, and optimize the operating conditions. ${ }^{1}$

Of various approaches, droplet-based bioprinting has proven to be a promising form of bioprinting due to its simplicity, high spatial resolution, high throughput, noncontact printing, and ability to form concentration gradients of bioactive materials. ${ }^{6}$ There are several droplet-based cell-printing modalities including inkjet bioprinting, $1,14,15$ acoustic droplet ejection, ${ }^{16,17}$ and microvalve bioprinting. ${ }^{18,19}$ Inkjet bioprinting, the most widely used technique for biological applications, is based on conventional inkjet printing technology. ${ }^{5}$ Inkjet bioprinters are generally classified as continuous and drop-on-demand inkjet printers. Drop-on-demand inkjet printing is particularly well-suited for deposition of living cells since it is designed to generate picoliter-scale droplets from aqueous solutions and precisely position them on a substrate; this approach can be used directly for the deposition of most biological species. ${ }^{3}$ However, inkjet bioprinting faces several challenges such as a narrow range of available bioinks, significant bioprinting-induced cell damage, limited mechanical and structural integrity of the bioprinted constructs, and restrictions on the size of the constructs due to a lack of vascularization and scaffold porosity. ${ }^{6}$ The cell damage mainly occurs 
during ejection and impingement of a cell-loaded droplet onto the substrate; the latter of these processes is the subject of the present study. In the nozzlefree methods, such as the acoustic bioprinting, ${ }^{16}$ impingement is the main cause of the cell damage. In addition, in the cell spray deposition method, the mechanical forces exerted on the cells within the spray nozzle are much lower than the shear stress that occurs during the impact on the substrate. ${ }^{20}$

Despite the cushioning provided by the encapsulating droplet, the cell may undergo significant deformation with large and rapid increases in the physical forces, such as shear stress and pressure, during the impact of the droplet on the substrate. This significantly influences not only the cell viability but also its ability to perform its essential functions in the tissue construct in the long term after printing. Therefore, these stresses can determine the success of the entire cell-printing process. The biomaterial solutions used to encapsulate living cells are called bioinks, and it is crucial that the selected bioink provides sufficient protection for the cells against stressors during the printing process. There are four main types of bioink materials: hydrogels, microcarriers, cell aggregates, and decellularized matrix components. ${ }^{21}$ All of these materials contain long-chain molecules (polymers) and hence exhibit viscoelastic behavior. In addition, biological cells naturally contain various long-chain molecules (proteins), making viscoelastic liquids inherently a better model for the cells compared to the Newtonian counterparts. ${ }^{22,23}$ Therefore, it is of fundamental importance to understand effects of viscoelasticity on the bioprinting process.

Cell viability, defined as the number of healthy cells in a sample, determines the amount of alive cells, based on a total cell sample. Viability assays are used to determine the ability of cells to maintain viability and it is quantified by an index between 0 and 1 . The value of 0 represents dead cells, while the value of 1 represents fully alive cells. Given the fact that the cell viability and functionality are closely related to the mechanical stresses exerted on the cell during the printing process, computational modeling can be used to gain deeper insight into impact dynamics of a cell-loaded droplet and thereby optimize cell-printing parameters such as the impact velocity, relative size of encapsulating droplet, and material properties of bioinks. ${ }^{13}$ Computational modeling of the droplet-based bioprinting is a challenging task due to highly complicated interactions between the cell, the encapsulating droplet, and the substrate. Adding viscoelasticity to the encapsulating droplet and the cell fluids makes the problem even more challenging for the numerical simulations. Early computational modeling of the cell-encapsulating droplet printing was performed by Wang et al. ${ }^{24}$ using a smoothed particle hydrodynamics method; however, this model was created under the assumption that the receiving substrate is coated with the same liquid as that of the encapsulating droplet. Tasoglu et al. ${ }^{25}$ used a compound-droplet model in which both the biological cell and the bioink are modeled as Newtonian fluids. Based on the cell viability model proposed by Takamatsu and Rubinsky, ${ }^{26}$ they hypothesized that cell damage is related to the increase in area of cell membrane. Therefore, they modeled the probability of cell survival as a function of the relative change in the cell membrane area compared to that of an undisturbed cell. They showed that high cell viability can be achieved by decreasing Reynolds number and the surface tension at the air-bioink interface. They also observed that cell viability increases as the equilibrium contact angle, cell/droplet viscosity ratio, and droplet/cell diameter ratio increase. Later, Hendriks et al. ${ }^{20}$ developed an analytical model based partly on the computational results of Tasoglu et al. ${ }^{25}$ and demonstrated that the cell viability can be controlled and optimized by manipulating the properties of the droplet fluid and operating conditions. He et al. ${ }^{27}$ modeled the droplet-based bioprinting as a compound droplet deposited into a pool of a Newtonian viscous solution in which the cell is assumed to be an elastic solid encapsulated by a viscous Newtonian fluid. Using this model, they identified four stages within the cell-printing process and provided an estimation for the duration and magnitude of the mechanical stresses exerted on the cell in each stage. In the present study, we use a compound-droplet model similar to that of Tasoglu et al. ${ }^{25}$ but we instead model the cell and the encapsulating droplet as viscoelastic liquids with different material properties. We thus focus on the effects of viscoelasticity on cell viability in the droplet-based bioprinting.

Compound droplets have generally been studied in the context of Newtonian liquid systems s $^{28-35}$ and only a few studies have considered the effects of viscoelasticity on the deformation and dynamics of compound droplets. ${ }^{36-38}$ Toose et al. ${ }^{36}$ developed a boundary element method to model the dynamics of non-Newtonian compound droplets under axisymmetric flow conditions. They identified the dominant breakup mechanism of compound drops in relation to the specific non-Newtonian behavior of the membrane. Zhou et al. ${ }^{37}$ computationally studied the formation of a viscoelastic compound droplet in a flow-focusing geometry but did not consider its dynamics afterward. More recently, Domejean et al. ${ }^{38}$ demonstrated the controlled production of submillimeter liquid core hydrogel capsules for multiplexed three-dimensional (3D) cell culture.

Viscoelastic compound droplets have often been used to model biological cells, e.g., white blood cells. ${ }^{39-41}$ Khismatullin and Truskey $^{39}$ numerically studied the effects of cell deformability and viscoelasticity on the adhesion of leukocytes to surfaces in a parallelplate flow chamber. They found that viscoelasticity has a significant effect on leukocyte adhesion. They demonstrated that cell viscoelasticity triggers the cell to transition from a spherical to a teardrop shape and thereby slowing down its rolling velocity significantly. Tatsumi et al. ${ }^{41}$ studied the dynamics of lymphocytes in a microchannel both numerically and experimentally. They modeled a lymphocyte using a non-Newtonian compound droplet with shearthinning effects in the cytoplasm and the nucleus. They found that the conventional Newtonian simple-droplet model fails to predict the deformation of a cell in the contraction region, where the nonlinear effects become significant. On the other hand, the compounddrop model yielded the results that were consistent with the experimental measurements of lymphocyte deformation. More recently, Nooranidoost et al. ${ }^{42}$ developed a compound-droplet model to study encapsulation of biological cells in a flow-focusing geometry. They developed a phase diagram for a range of inner and outer capillary numbers, identified four different cell encapsulation modes and determined the conditions for the single cell encapsulation as the favorable mode for bioprinting systems. They also examined the effect of viscoelasticity on formation of cell-encapsulating droplets. ${ }^{43}$ They found that success rate for the single cell encapsulation may be improved by adjusting viscoelasticity of the shell fluid. 
In the present study, for the first time, we computationally investigate the effects of viscoelasticity on the dynamics of a compound droplet impacting on a flat substrate as a model for the droplet-based bioprinting. The compound droplet model consists of the inner droplet (cell), the encapsulating droplet (bioink), and the surrounding air. The cell and bioink are modeled as viscoelastic fluids with different material properties while air is modeled as a Newtonian fluid. The receiving substrate is modeled as a perfectly smooth and rigid wall. The viscoelasticity is accounted for using the FENE-CR model (Finite Extendable Nonlinear Elastic-Chilcott and Rallison $)^{44}$ and particle-resolved simulations are performed using a front-tracking method for a wide range of viscoelastic parameters. In particular, extensive computations are performed to investigate the cell deformation (and, indirectly, the cell viability) as a function of the Weissenberg number $\left(W i_{d}\right)$ and the polymeric viscosity ratio $\left(\beta_{d}\right)$ based on the encapsulating droplet fluid properties in the ranges of $0 \leq W i_{d} \leq 100$ and $0 \leq \beta_{d} \leq 0.9$. Further simulations are also carried out to demonstrate the importance of the cell viscoelasticity for accurate predictions of the cell viability.

\section{PROBLEM STATEMENT AND FORMULATION}

The impingement of a compound droplet on a flat surface is shown schematically in Fig. 1, where the cell, the bioink, the surrounding air, and the solid substrate are shown in red, green, blue, and yellow, respectively. The compound droplet is initially located close to the flat surface and initiated with a uniform impact velocity $\left(V_{c o l}\right)$ directed toward the substrate [Fig. 1(a)]. The ambient fluid is initially quiescent. The compound droplet impinges and spreads on the solid surface, which causes the cell to deform due to the stresses built up in the bioink [Fig. 1(b)]. This deformation is the main cause for the cell damage that may result in its death in extreme cases. Both the cell and bioink fluids contain long-chain molecules and thus exhibit viscoelastic behavior. The effects of viscoelasticity contained in the cell and bioink on the mechanical deformation and viability of the cell under different conditions of practical importance are our main focus in the present study. For this purpose, the cell and the encapsulating droplet are modeled as viscoelastic fluids using a FENE-CR model, ${ }^{44}$ while the ambient fluid (air) is modeled as a Newtonian fluid. In a FENE-CR liquid, polymers are modeled as finitely extensible mass-spring dumbbells in a solution, so it is deemed to be a more realistic than the Maxwell and Oldroyd-B models where the polymers are assumed to be infinitely extensible. ${ }^{36,37}$ The substrate is treated as a rigid solid with a smooth flat surface. The flow is assumed to be axisymmetric. Therefore, only one half is used as the computational domain that extends 3 and 1.5 encapsulating droplet diameters in the radial and the axial directions, respectively. The computational domain and the symmetry axis are, respectively, shown by the white and gray dashed lines in Fig. 1(b). Symmetry boundary conditions are applied at the axis of symmetry while the gradient-free boundary conditions are imposed at the top and lateral boundaries for all the flow quantities. The no-slip boundary conditions are used on the solid wall for all the flow quantities except for the interface for which the no-slip boundary conditions are known to yield a stress singularity near the contact line. The boundary conditions are expressed mathematically in Fig. 1(b), where $u, v, \mathbf{A}$, and $p$ are the velocity components in the radial and axial directions, conformation tensor and pressure, respectively.

Following Muradoglu and Tasoglu, ${ }^{57}$ a slip-contact line model based on the dynamic contact angle is used to treat the moving contact line. The treatment is briefly described here for completeness and the readers are referred to Muradoglu and Tasoglu ${ }^{57}$ for the details. The drop interface is monitored during the simulation and the front-element crossing the threshold line (e.g., the horizontal dashed magenta line in the sketch) is connected to the wall such that the contact angle is equal to the dynamic contact angle of $\theta_{D}$. Kistler's correlation ${ }^{58}$ is used to relate the dynamic contact angle to the static contact angle $\left(\theta_{e}\right)$ and the capillary number $C a_{c l}=\mu_{d}\left|V_{c l}\right| / \sigma_{o}$, where $\mu_{d}, V_{c l}$, and $\sigma_{o}$ are viscosity of the encapsulating droplet, velocity of the contact line and interfacial tension of the outer interface, respectively. To do this, we first compute

$$
\theta_{D_{i}}=f_{\text {Hoff }}\left(C a_{c l}+f_{\text {Hoff }}^{-1}\left(\theta_{e}\right)\right),
$$

where $f_{\text {Hoff }}$ is the Hoffman function defined as

$$
f_{\text {Hoff }}(\xi)=\arccos \left\{1-2 \tanh \left[5.16\left(\frac{\xi}{1+1.31 \xi^{0.99}}\right)^{0.706}\right]\right\} .
$$

We finally compute the dynamic contact angle as

$$
\theta_{D}=\left\{\begin{array}{cll}
\theta_{D_{i}} & \text { if } V_{c l} \geq 0 & \text { (advancing), } \\
2 \theta_{e}-\theta_{D_{i}} & \text { if } V_{c l}<0 & \text { (receding). }
\end{array}\right.
$$

The implementation details of the slip-contact line model can be found in Muradoglu and Tasoglu.

The deformation and the rate of deformation of the inner droplet (cell) are hypothesized to determine the viability of the cell. ${ }^{25}$

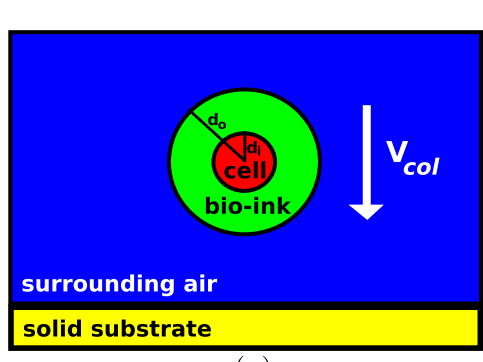

(a)

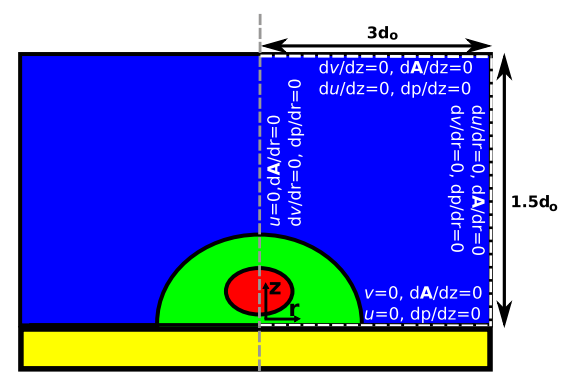

(b)
FIG. 1. Schematic illustration of the impingement and spreading of a compound droplet on a solid substrate in a bioprinting system. (a) The initial position and (b) after the impingement. 
The deformation of the cell is quantified using the deformation parameter defined as $\gamma=A / A_{0}$, where $A$ and $A_{0}$ are the surface areas of the deformed and the undeformed cells, respectively. ${ }^{20,26}$ We also use a cell viability model proposed by Takamatsu and Rubinsky ${ }^{26}$ to compute the cell viability and to identify how much the cell is damaged due to mechanical deformation. This model is based on the experimental data obtained from compression of cells between two parallel plates. They measured the percentage of destroyed cells by calculating the ratio between the total number of cells and impaired cells stained by trypan blue. Then, assuming the cells experience a geometrically ideal deformation, they derived a correlation between relative change in the cell membrane area and cell destruction. ${ }^{26}$ As a cell deforms, its surface area increases proportionally. Takamatsu and Rubinsky ${ }^{26}$ found that the cell membrane is stretched but the cell remains intact until the cell membrane area increases up to $5 \%$. However, a further increase in the cell surface area can result in a rupture and consequently death of the cell. Then, they modeled the probability of cell survival $(\eta)$ as a function of maximum instantaneous value of cell deformation $\left(\gamma_{\max }\right)$ and proposed the following relation for the viability of an individual cell:

$$
\eta(\gamma)= \begin{cases}1 & \text { for } \gamma_{\max }<\gamma_{c r}-\Delta \gamma \\ \frac{1}{2}-\frac{\gamma_{\max }-\gamma_{c r}}{2 \Delta \gamma} & \text { for } \gamma_{c r}-\Delta \gamma \leq \gamma_{\max } \leq \gamma_{c r}+\Delta \gamma \\ 0 & \text { for } \gamma_{\max }>\gamma_{c r}+\Delta \gamma\end{cases}
$$

where the critical membrane expansion (critical cell deformation) and the range of surface expansion in which the cells partly survive are specified as $\gamma_{c r}=1.5$ and $\Delta \gamma=0.5$, respectively. Hence, the probability of cell survival $(\eta)$ varies between $0 \leq \eta \leq 1$, it is 0 when $\gamma_{\max }>2$ (i.e., the cell is dead) and is 1 when $\gamma_{\max } \leq 1$ (i.e., the cell is certainly alive). An important deficiency of this model is that it does not say anything about the loss of cell functionality caused by mechanical stress and deformation. This model assumes an elastic cell membrane response to mechanical stresses and negligible lipid membrane replenishment during the deformation. ${ }^{20,2}$ We also note that this model assumes a perfectly rigid substrate and thus underpredicts the cell viability for the case where the substrate is soft. ${ }^{20}$

Assuming that the flow is incompressible in all phases, the mass and momentum conservation equations can be written as ${ }^{46,47}$

$$
\nabla \cdot \mathbf{u}=0
$$

$$
\begin{aligned}
\frac{\partial \rho \mathbf{u}}{\partial t}+\nabla \cdot(\rho \mathbf{u u})= & -\nabla p+\nabla \cdot \mu_{s}\left(\nabla \mathbf{u}+\nabla \mathbf{u}^{T}\right)+\nabla \cdot \boldsymbol{\tau} \\
& +\int_{A} \sigma \kappa \mathbf{n} \delta\left(\mathbf{x}-\mathbf{x}_{\mathbf{f}}\right) d A
\end{aligned}
$$

where $\mathbf{u}$ is the velocity vector, $\rho$ and $\mu_{s}$ are the discontinuous density and solvent viscosity fields, respectively, $p$ is the pressure, and $\tau$ is the viscoelastic extra stress tensor. The last term in the momentum equations represents the surface tension, where $\sigma$ is the surface tension coefficient, $\kappa$ is the mean curvature, $\mathbf{n}$ is the outward unit vector normal to the interface, and $\delta$ is the three-dimensional delta function. The surface tension force acts only on the interface location denoted by $\mathbf{x}_{\mathbf{f}}$.

Both the cell and encapsulating fluids are modeled as viscoelastic liquids using the FENE-CR model ${ }^{44}$ given by

$$
\begin{gathered}
\frac{\partial \mathbf{A}}{\partial t}+\nabla \cdot(\mathbf{u A})-(\nabla \mathbf{u})^{T} \cdot \mathbf{A}-\mathbf{A} \cdot \nabla \mathbf{u}=-\frac{L^{2}}{L^{2}-\operatorname{trace}(\mathbf{A})} \frac{\mathbf{A}-\mathbf{I}}{\lambda}, \\
\boldsymbol{\tau}=\mu_{p}\left(\frac{L^{2}}{L^{2}-\operatorname{trace}(\mathbf{A})}\right) \frac{\mathbf{A}-\mathbf{I}}{\lambda},
\end{gathered}
$$

where $\mathbf{A}, \lambda, L, \mathbf{I}$, and $\mu_{p}$ are the conformation tensor, the relaxation time, the extensibility parameter (i.e., the ratio of the length of a fully extended polymer dumbbell to its equilibrium length), the identity tensor, and polymeric viscosity, respectively. Note that the conformation tensor (A) is a symmetric positive definite tensor representing the average conformation of coil structure of polymers under different flow conditions. ${ }^{45}$ The conformation tensor may be considered as the nondimensional viscoelastic stress tensor.

In the simulations, the diameters of the encapsulating droplet and the cell are set to the experimental values of Demirci and Montesano $^{16}$ as $d_{o}=37 \mu \mathrm{m}$ and $d_{i}=13 \mu \mathrm{m}$, respectively. The density and the total viscosity of the encapsulating droplet and the cell are selected to be in the range of the values used by Tasoglu et al. ${ }^{25}$ and are summarized in Table I for all the three phases of the inner droplet (cell), the encapsulating droplet (bioink), and the ambient air. Note that the total viscosity is defined as the sum of the solvent and polymeric viscosities, i.e., $\mu=\mu_{s}+\mu_{p}$. These values are also in the range of the parameters used by Hendriks et al. ${ }^{20}$ except for the viscosity of the cell and the density of the ambient fluid. The viscosity of the cell is taken here as an order of magnitude larger than that of the encapsulating droplet mainly for the numerical purposes although the actual apparent viscosity of the cell is much higher. For the same reason, the density of the surrounding air is also set to $\rho_{o}=51.5 \mathrm{~kg} / \mathrm{m}^{3}$, which is about 40 times larger than its physical value. Although not shown here, it is found that the density of ambient fluid does not have any significant influence on the impact dynamics as long as it is 20 times or more smaller than that of the encapsulating droplet liquid as also reported by Tasoglu et al. ${ }^{25}$ Surface tension at the air-bioink interface and the bioink-cell interface is 0.07622 and $0.00003 \mathrm{~N} / \mathrm{m}$, respectively $\left(\sigma_{o} / \sigma_{i}=2541\right)$, as also used by Tasoglu et al. ${ }^{25}$ Note that the same value for the surface tension at the air-bioink was also used by Matubayasi and Nishiyama. ${ }^{59}$ Unless specified otherwise, in all the results presented in this paper, the relaxation time and the polymeric viscosity ratio for the cell are specified as $\lambda_{c}=1.85 \times 10^{-3} \mathrm{~s}$ and $\beta_{c}=0.5$, respectively. We selected these values to get the Weissenberg number in the range $0 \leq W i \leq 100$, which is in the range of previous numerical studies. ${ }^{37,60}$ The relaxation time and the polymeric viscosity ratio for the encapsulating droplet are varied between zero (Newtonian)

TABLE I. The material properties of the ambient, the encapsulating droplet, and the cell fluids.

\begin{tabular}{lccc}
\hline \hline Fluids & $\begin{array}{c}\text { Density } \\
\left(\mathrm{kg} / \mathrm{m}^{3}\right)^{25}\end{array}$ & $\begin{array}{c}\text { Total viscosity } \\
(\mathrm{Pa} \mathrm{s})^{25}\end{array}$ & $\begin{array}{c}\text { Relaxation } \\
\text { time }(\mathrm{s})\end{array}$ \\
\hline Ambient fluid & 51.5 & $6.35 \times 10^{-5}$ & 0 \\
Encapsulating droplet & 1030 & $2.54 \times 10^{-3}$ & $0-1.85 \times 10^{-3}$ \\
Cell & 1030 & $1.27 \times 10^{-2}$ & $1.85 \times 10^{-3}$ \\
\hline \hline
\end{tabular}


and the respective value of the cell fluid. Following Izbassarov and Muradoglu, ${ }^{51}$ the extensibility parameter for the cell and the encapsulating droplet fluids is assumed to be the same and specified as $L=15$. Although not shown here, we also performed simulations for various values of the extensibility parameter and found that this parameter does not affect the cell deformation and viability significantly. The equilibrium contact angle is specified as $\theta_{e}=60^{\circ}$, which is much larger than the value in the experimental study, i.e., the static contact angle in the experimental work is around $10^{\circ} .{ }^{16} \mathrm{We}$ choose a larger contact angle because it is computationally expensive to resolve the thin liquid layer close to the solid surface for small contact angles and, more importantly, a higher contact angle (or hydrophobicity) is more desirable to prevent cell damage. In fact, in many bioprinting systems, a thin layer of coating material such as 3-(Trimethoxysilyl)propyl methacrylate (TMSPMA) is usually used to coat the substrate in order to enhance the hydrophobicity before printing. ${ }^{61,62}$ This coating will increase the contact angle and the ultimate contact angle is typically in the range of $45^{\circ} \leq \theta_{e} \leq 135^{\circ}$. Therefore, our equilibrium contact angle of $60^{\circ}$ is an accepted value. The impact velocity is specified as $V_{c o l}=2 \mathrm{~m} / \mathrm{s}$ in all the results presented in this paper as a representative value of the experimental conditions. $^{16}$

The governing equations are solved in their dimensional forms but the results are expressed in terms of the relevant nondimensional parameters. The length and the velocity scales are selected as the initial droplet diameter $\left(d_{o}\right)$ and the impact velocity $\left(V_{c o l}\right)$, respectively. Time scale is then computed as $d_{o} / V_{c o l}$. The pressure and stress components are normalized with $\rho V_{c o l}^{2}$. Based on these scales, the relevant nondimensional parameters can be written as

$$
\begin{gathered}
W i_{c}=\frac{\lambda_{c} V_{c o l}}{d_{o}}, W i_{d}=\frac{\lambda_{d} V_{c o l}}{d_{o}}, W e=\frac{\rho_{d} V_{c o l}^{2} d_{o}}{\sigma_{o}}, \\
R e=\frac{\rho_{d} V_{c o l} d_{o}}{\mu_{d}}, \frac{\rho_{d}}{\rho_{o}}, \frac{\rho_{c}}{\rho_{d}}, \beta_{d}=\frac{\mu_{p, d}}{\mu_{p, d}+\mu_{s, d}}, \beta_{c}=\frac{\mu_{p, c}}{\mu_{p, c}+\mu_{s, c}}, \\
\frac{\mu_{d}}{\mu_{o}}=\frac{\mu_{p, d}+\mu_{s, d}}{\mu_{o}}, \frac{\mu_{c}}{\mu_{d}}=\frac{\mu_{p, c}+\mu_{s, c}}{\mu_{p, d}+\mu_{s, d}},
\end{gathered}
$$

where $W i_{c}, W i_{d}, W e, R e, \beta_{d}$, and $\beta_{c}$ are the Weissenberg number of the cell, the Weissenberg number of the encapsulating droplet, the Weber number, the Reynolds number, the polymeric viscosity ratios in the encapsulating droplet, and in the cell, respectively.

\section{NUMERICAL METHOD}

A one-field formulation is used to solve the flow equations for all phases in the entire computational domain. The flow equations [Eqs. (5) and (6)] are solved fully coupled with the viscoelastic model equations [Eqs. (7) and (8)] in two steps using a projection method. In the first step the pressure gradient is ignored and the unprojected velocity is computed. Then, using this unprojected velocity the pressure is calculated by solving a nonseparable Poisson equation.

We use the front-tracking method developed for particleresolved simulations of viscoelastic multiphase flow systems by Izbassarov and Muradoglu ${ }^{48}$ to treat the multiphase part of our simulations. The front-tracking method was introduced by Unverdi and Tryggvason ${ }^{46}$ and has been successfully applied to study a wide range of interfacial flows as reviewed by Tryggvason et al. ${ }^{47,49}$ The method has been extended to treat viscoelastic effects and applied to study various multiphase flows. ${ }^{50-54}$ In a front-tracking method, the field equations are solved on a regular structured staggered Eulerian grid using a projection method and the interface between different phase is tracked explicitly by a Lagrangian grid consisting of connected marker points ${ }^{47}$ as shown in Fig. 2. The marker points are advected with the local flow velocity interpolated from the Eulerian grid and then the indicator function is computed based on the location of the marker points using the standard procedure as described by Tryggvason et al. ${ }^{47}$ The Lagrangian grid (marker points) are also used to compute surface tension, which is then smoothed on to the Eulerian grid and added to the discrete momentum equations [Eq. (6)]. As the interface evolves with the flow, the marker points are dynamically added or deleted to keep the point density nearly uniform and comparable to the underlying Eulerian grid, which is needed to fully resolve the interface.

The material properties vary discontinuously across phase boundaries. An indicator function, $\phi$, is used to distinguish different phases and to set the material properties in the entire computational domain. Referring to Fig. 2, the indicator function is defined for the present three-phase flow as

$$
\phi=\left\{\begin{array}{l}
2 \text { in the cell, } \\
1 \text { in the encapsulating droplet, } \\
0 \text { in the ambient fluid. }
\end{array}\right.
$$

The indicator function can be computed using the standard technique based on the location of the interfaces as described by Tryggvason et al. ${ }^{47}$ Once it is computed, any generic material property, $\psi$, can be set in all three phases as

$$
\psi= \begin{cases}\psi_{d} \phi+\psi_{o}(1-\phi) & \text { if } \phi \leq 1, \\ \psi_{c}(\phi-1)+\psi_{d}(2-\phi) & \text { otherwise }\end{cases}
$$

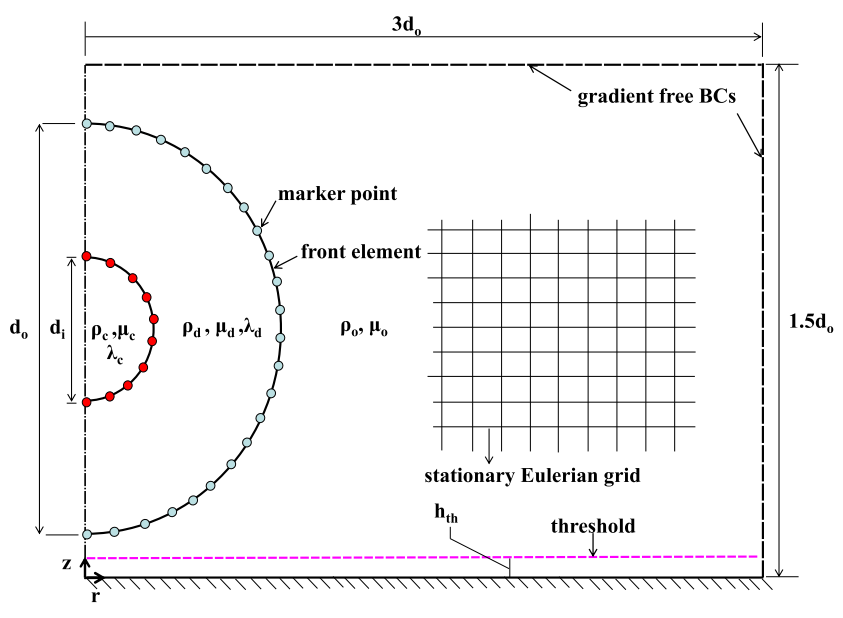

FIG. 2. Schematic illustration of the computational setup. The interfaces are represented by a Lagrangian grid consisting of marker points and the field equations are solved on a stationary Eulerian grid. 
where the subscripts $c, d$, and $o$ denote the properties of the cell liquid, the encapsulating droplet liquid, and the ambient fluid, respectively. Equation (11) is used to compute density $\rho$, solvent viscosity $\mu_{s}$, polymeric viscosity $\mu_{p}$, and relaxation time $\lambda$ in the entire computational domain.

The spatial derivatives are approximated using central differences on a staggered Eulerian grid except for the convective terms in the viscoelastic model equations for which a fifth-order upwind WENO-Z scheme ${ }^{55}$ is used to resolve a thin viscoelastic stress boundary layer especially near the fluid-fluid interfaces. Time integration is performed using a first order explicit Euler method. Although not shown here, it is generally found that the temporal error is negligible and much smaller than the spatial error in all the results presented in this paper. The time step is restricted to maintain numerical stability of the present explicit method. Considering the viscous, convective, and capillary time scales, the time step is determined based on the linear stability as

$$
\Delta t=\alpha_{s f} \min \left(\frac{h_{\min }^{2}}{4 s_{\max }}, \frac{h_{\min }}{U_{\max }}, \sqrt{\frac{\left(\left(\rho_{c}+\rho_{d}\right) h_{\min }^{3}\right)}{4 \pi \sigma_{i}}}, \sqrt{\frac{\left(\left(\rho_{d}+\rho_{o}\right) h_{\min }^{3}\right)}{4 \pi \sigma_{o}}}\right),
$$

where $s_{\text {max }}$ is the largest among the kinematic viscosities of the fluids $\mu_{c} / \rho_{c}, \mu_{d} / \rho_{d}$ and $\mu_{o} / \rho_{o}, h_{\text {min }}$ is the smallest grid size and $U_{\max }$ is the maximum magnitude of the velocity in the domain. Note that the time step is multiplied by a safety factor, $\alpha_{s f}$, taken here as $\alpha_{s f}=0.425$ in order to avoid instabilities due to viscoelastic and other nonlinear effects. We note that a second-order predictorcorrector time integration scheme is readily available as an option in the present numerical method but the first order scheme is used to facilitate extensive simulations.

The log-conformation method developed by Fattal and Kupfer$\operatorname{man}^{56}$ is employed to circumvent the high Weissenberg number problem (HWNP) in solving the viscoelastic model equations. As discussed by Izbassarov and Muradoglu, ${ }^{48,50,51}$ the log-conformation method preserves the positive-definiteness of the conformation tensor at the numerical solution level and thus stabilizes the numerical scheme even at very large values of the Weissenberg numbers.

Finally, for the contact line treatment, the drop interface is assumed to connect the solid wall when the distance between the drop and solid wall becomes smaller than a prespecified threshold value $h_{t h}$ (Fig. 2), taken in the present study as $h_{t h}=2 \Delta z$ where $\Delta z$ is the Eulerian grid size. We note that Muradoglu and Tasoglu showed that the results are not very sensitive to the threshold distance in the range $1.5 \Delta z \leq h_{t h} \leq 3 \Delta z$. The details of numerical methods for the front-tracking method, viscoelasticity, and treatment of contact line can be found in Tryggvason et al., ${ }^{47}$ Izbassarov and Muradoglu, ${ }^{48}$ and Muradoglu and Tasoglu, ${ }^{57}$ respectively.

Simulations are performed by varying a single parameter at a time while keeping all the other parameters constant in order to demonstrate the sole effects of the given parameter on the flow. To facilitate this, we define a baseline case as

$$
\begin{gathered}
W i_{c}=100, W i_{d}=1, W e=2, \operatorname{Re}=30, \beta_{d}=0.5, \\
\beta_{c}=0.5, L=15, \sigma_{o} / \sigma_{i}=2541, \frac{\mu_{c}}{\mu_{d}}=5, \frac{\mu_{d}}{\mu_{o}}=40, \\
\frac{\rho_{d}}{\rho_{o}}=20, \frac{\rho_{c}}{\rho_{d}}=1, d_{o} / d_{i}=2.85, \theta_{e}=60^{\circ} .
\end{gathered}
$$
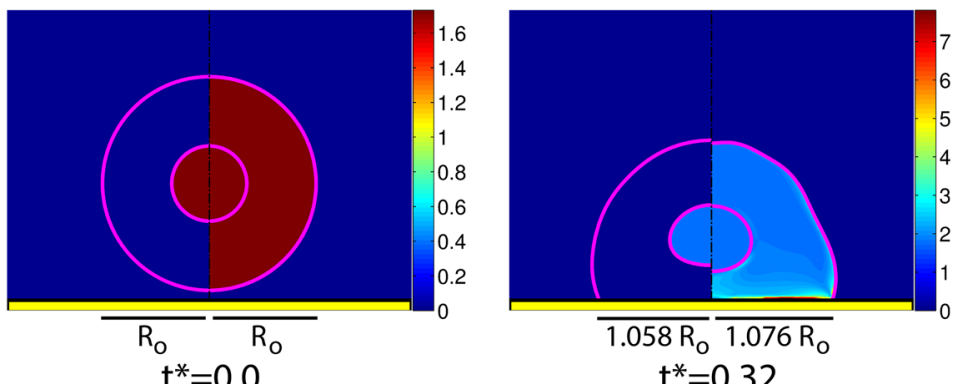

$\overline{1.058 \mathrm{R}_{\mathrm{o}}} \overline{1.076 \mathrm{R}_{\mathrm{o}}}$ $t^{*}=0.32$

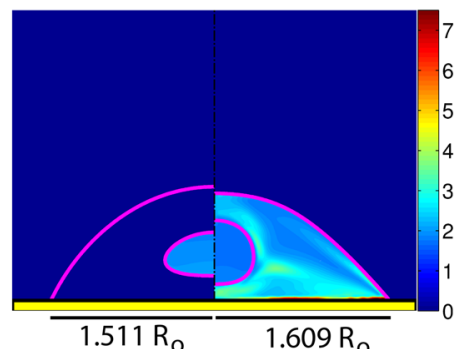

$t^{*}=1.081$

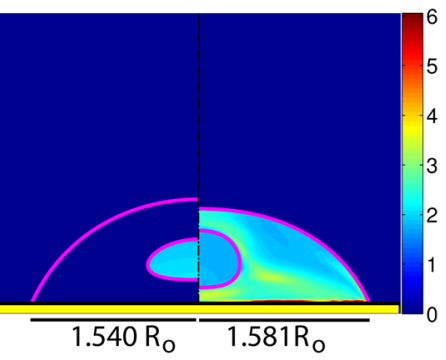

$t^{*}=1.621$

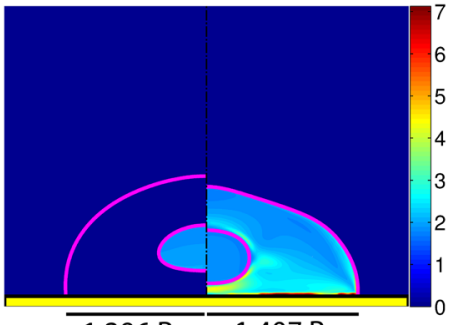

$1.296 \mathrm{R}_{\mathrm{o}} \quad 1.407 \mathrm{R}_{\mathrm{o}}$ $t^{*}=0.54$

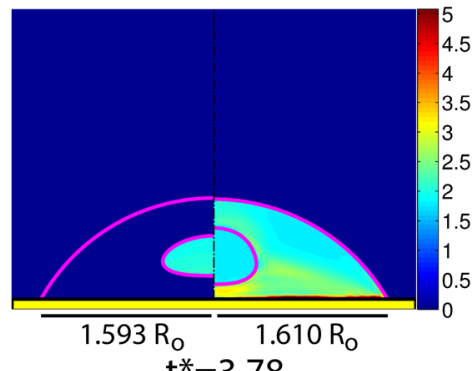

$t^{*}=3.78$

FIG. 3. Evolution of a compound droplet impacting on a flat surface. The left and right haves show the Newtonian $\left(W i_{d}=0\right)$ and the viscoelastic $\left(W i_{d}=10, \beta_{d}=0.9, L=15\right)$ encapsulating droplet cases, respectively. The extend of the encapsulating droplet is indicated by the thick horizontal black line together with the numerical value at each instant just below the corresponding inset, where $R_{0}$ is the radius of the initial encapsulating droplet. Time evolves from left to right and from top to bottom. $(W e=2, R e=30$, $W i_{c}=100, \beta_{c}=0.5, d_{0} / d_{i}=2.85, \sigma_{o} / \sigma_{i}=2541, \mu_{c} / \mu_{d}=5, \mu_{d} / \mu_{0}=40, \rho_{c} / \rho_{d}=1, \rho_{d} / \rho_{0}=20, \theta_{e}=60^{\circ}$.) 
Note that the baseline case is determined to be in the range of the parameters used in Tasoglu et al. ${ }^{25}$ Since we mainly focus here on the effects of the viscoelasticity contained in the bioinks, we present extensive simulation results showing the effects of the Weissenberg number and the polymeric viscosity ratio based on the encapsulating droplet fluid properties. However, we also present further results to demonstrate the importance of the cell viscoelasticity for accurate predictions of the cell viability.

\section{RESULTS AND DISCUSSIONS}

Extensive simulations are performed using the compound droplet model to investigate the effects of viscoelasticity of the encapsulating droplet on the cell viability during the impingement on a flat substrate. The viscoelasticity is characterized by the Weissenberg number and the polymeric viscosity ratio. Since previous studies have shown that cell damage is largely caused by cell
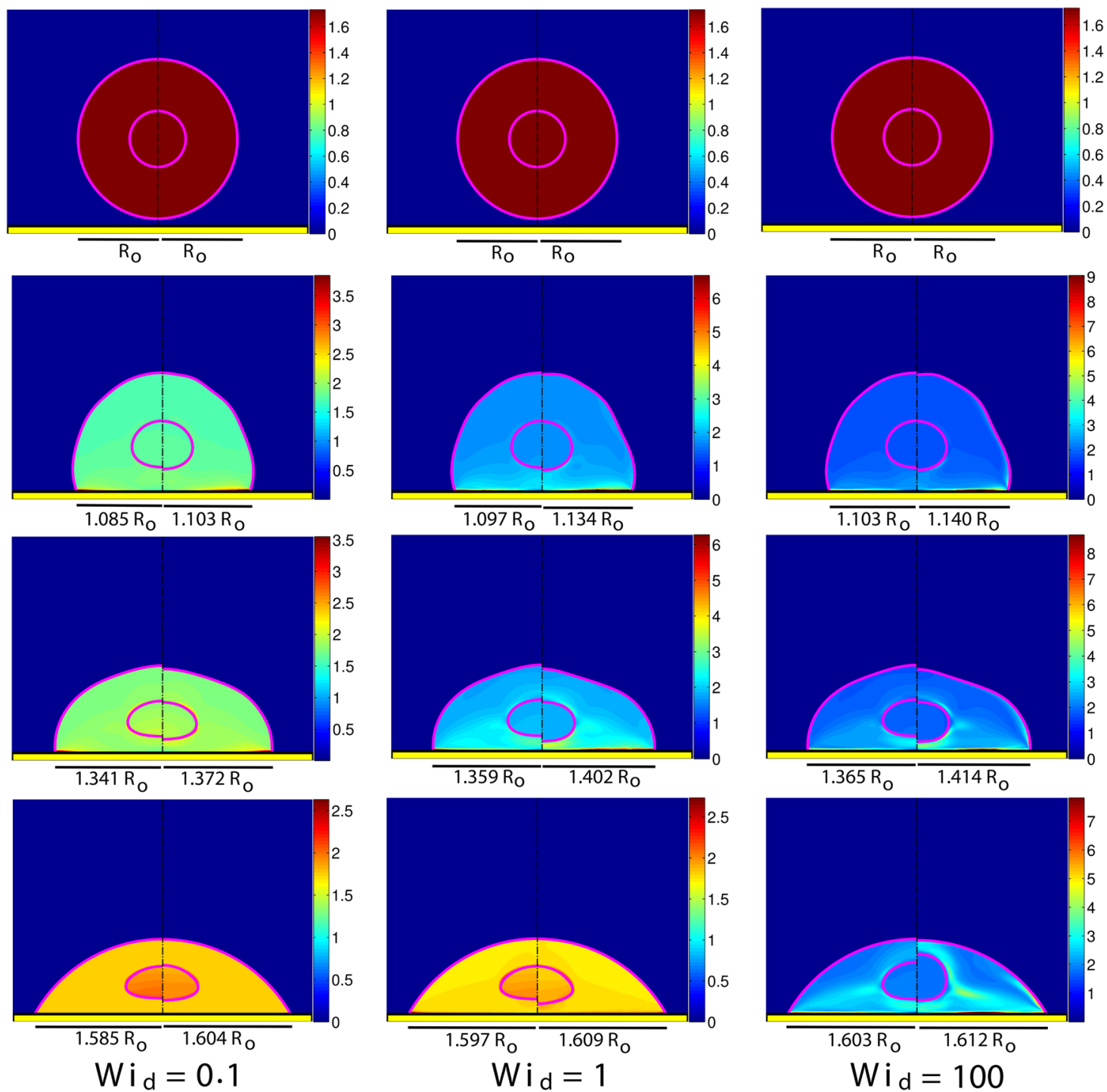

FIG. 4. Evolution of a viscoelastic compound droplet impacting on a flat substrate for a moderate polymeric viscosity ratio of $\beta_{d}=0.5$ (left half) and a high polymeric viscosity ratio of $\beta_{d}=0.9$ (right half), and for $W i_{d}=0.1,1$, and 100 from left to right, respectively. The extend of the encapsulating droplet is indicated by the thick horizontal black line together with the numerical value at each instant just below the corresponding inset, where $R_{0}$ is the radius of the initial encapsulating droplet. The time progress from top to bottom and the snapshots are taken at times $t^{*}=0,0.32,0.54$, and 3.78 . The constant contours denote the average polymer extension $[\sqrt{\operatorname{trace}(\mathbf{A})}] \cdot\left(W e=2, R e=30, W i_{c}=100, \beta_{c}=0.5, L=15, d_{0} / d_{i}=2.85, \sigma_{0} / \sigma_{i}=2541, \mu_{c} / \mu_{d}=5, \mu_{d} / \mu_{0}=40, \rho_{c} / \rho_{d}=1, \rho_{d} / \rho_{0}=20\right.$, $\theta_{e}=60^{\circ}$.) 
deformation rather than other stressors such as shear stress and pressure, ${ }^{20,25,26}$ we investigate the effect of viscoelasticity on cell deformation and relate it to cell viability.

A uniform grid is employed in all the computations. Although not shown here due the space considerations, a comprehensive grid convergence study is done to determine the minimum grid size required to reduce the spatial discretization error below a threshold value. It is found that a computational grid containing $512 \times 256$ cells in the radial and axial directions, respectively, is sufficient to reduce the spatial error below $0.5 \%$ for all the flow quantities. Therefore, this grid resolution is used in all the results presented in this paper. Since the time step is restricted to maintain the numerical stability in the present explicit numerical method, the time stepping error is found to be negligible compared to the spatial error as also reported in the previous studies. ${ }^{25,51,53}$ We also note that the code has been rigorously validated previously for the similar problems $^{25,51,57}$ so separate validation tests have not been repeated here.

We first show the effects of viscoelasticity of the encapsulating droplet fluid qualitatively in Fig. 3 where the snapshots taken at times $t^{*}=0,0.32,0.54,1.08,1.62$, and 3.78 are plotted for the Newtonian $\left(W i_{d}=0\right)$ and the viscoelastic $\left(W i_{d}=10\right.$ and $\left.\beta_{d}=0.9\right)$ encapsulating droplet cases on the left and the right sides, respectively. In both cases, the cell is viscoelastic ( $W i_{c}=100$ and $\left.\beta_{c}=0.5\right)$ and the other parameters are the same as the baseline case. The square root of the trace of the conformation tensor $[\sqrt{\operatorname{trace}(\mathbf{A})}]$ is also plotted in Fig. 3 as a measure of the average polymer extension. It is clearly seen in this figure that the cell
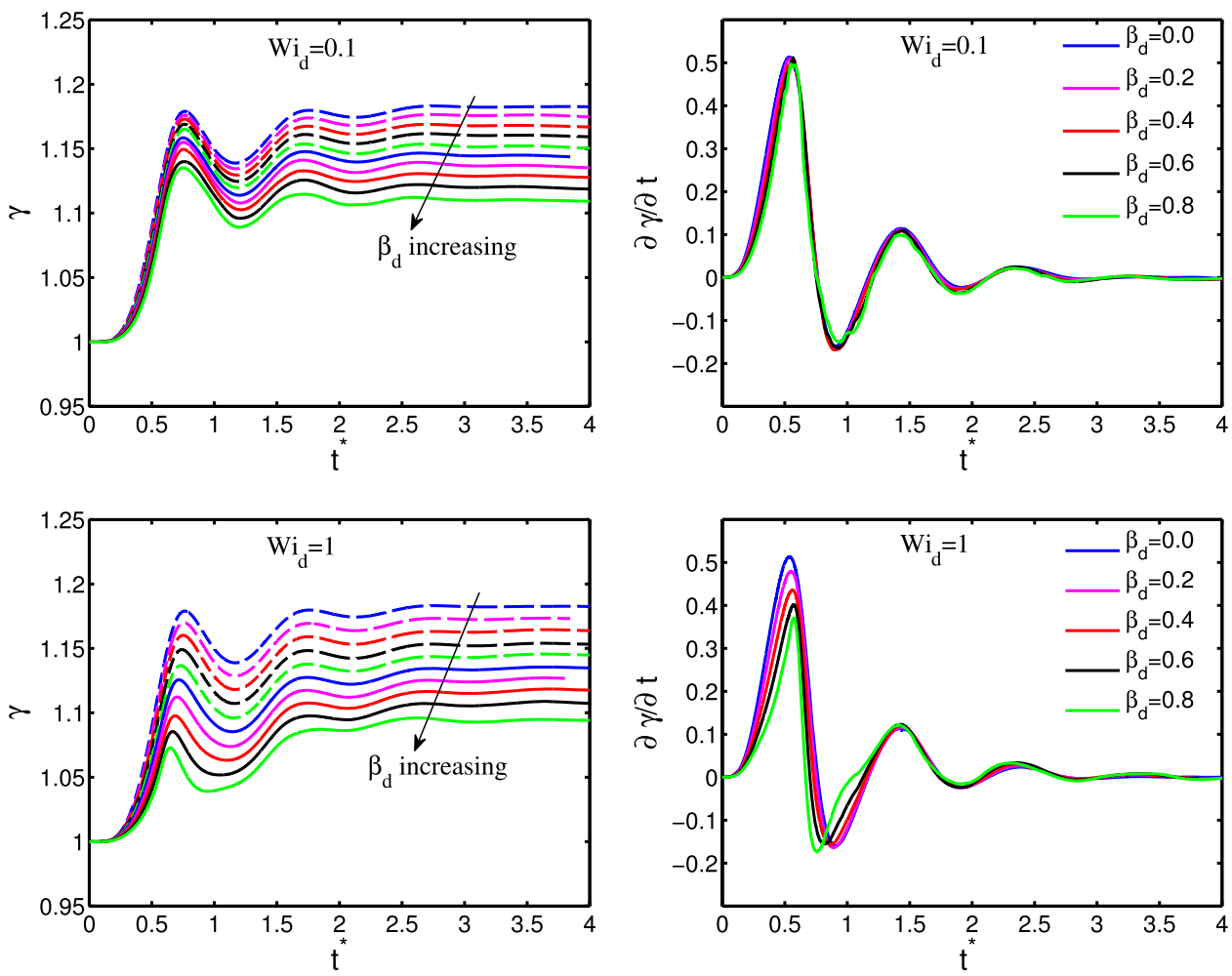

FIG. 5. Cell deformation (left) and rate of cell deformation (right) for $0 \leq \beta_{d} \leq 0.9$ and $W_{i_{d}}=0.1,1,100$. $(W e=2, R e=30$, $W i_{c}=100, \beta_{c}=0.5, L=15, d_{0} / d_{i}=2.85$ $\sigma_{0} / \sigma_{i}=2541, \mu_{c} / \mu_{d}=5, \mu_{d} / \mu_{0}=40$ $\rho_{c} / \rho_{d}=1, \rho_{d} / \rho_{0}=20, \theta_{e}=60^{\circ}$.)
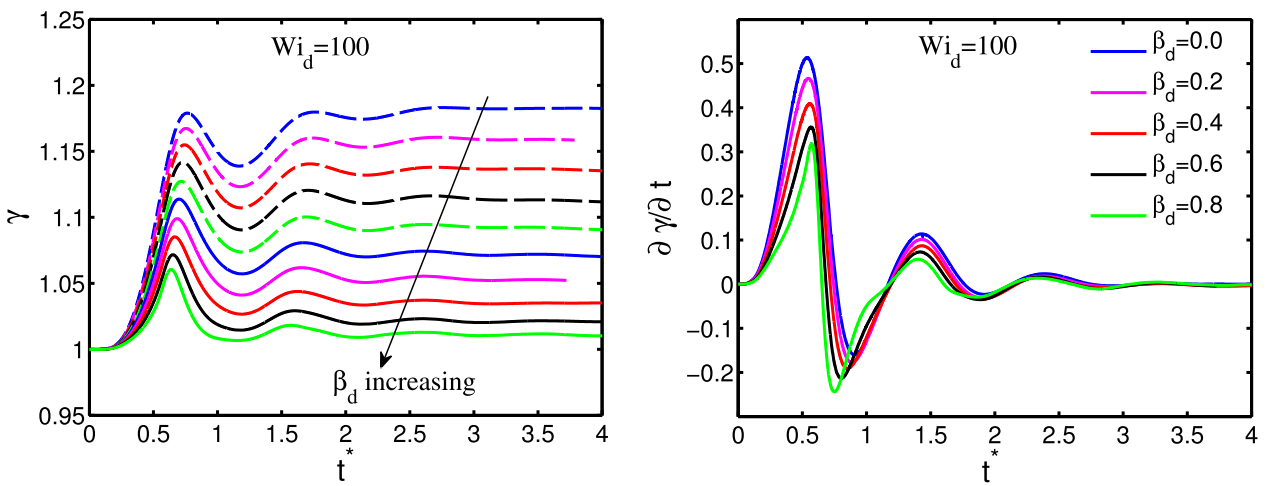
deformation is significantly reduced in the viscoelastic case showing the enhancement of the cell viability by the viscoelasticity of the bioinks. The extend of the encapsulating droplet is indicated by the thick horizontal black line together with the numerical value at each instant just below the corresponding inset, where $R_{o}$ is the radius of the initial encapsulating droplet. As the initially spherical compound droplet impacts and spreads on the substrate, the polymers in the viscoelastic encapsulating droplet are extended rapidly creating a thin viscoelastic boundary layer near the substrate. Although there is a large viscoelastic stress built up in the viscoelastic boundary layer, the cell is lubricated and kept sufficiently far from the wall as seen in the figure. It is interesting to observe that the deformation patterns are markedly different in the Newtonian and the viscoelastic cases. Although the encapsulating droplet deforms more, the inner droplet (cell) deforms significantly less in the viscoelastic case. A close examination reveals that the extended polymers in the boundary layer induce large viscoelastic stresses that push the fluid outward near the contact line to enhance the spreading rate. This is the main mechanism that makes the viscoelastic droplet spread more rapidly than that of the Newtonian counterpart as also reported by Izbassarov and Muradoglu. ${ }^{51}$ As a result, the fluid between the inner droplet and the wall drains more rapidly pulling the entire inner droplet down nearly uniformly, which significantly reduces the cell deformation and consequently improves its viability in the viscoelastic case.

We next investigate the effects of the viscoelastic parameters on the dynamics of the compound droplet and the cell viability. For this purpose, simulations are performed to examine the effects of the Weissenberg number of the encapsulating droplet by varying $W i_{d}$ in the range of $0.1 \leq W i_{d} \leq 100$ at a moderate $\left(\beta_{d}=0.5\right)$ and a high $\left(\beta_{d}=0.9\right)$ polymeric viscosity ratios, while the other parameters are fixed at the baseline values. The evolution of the droplet and the cell shapes are shown in Fig. 4 for $\beta_{d}=0.5$ (left half) and $\beta_{d}=0.9$ (right half), for $W i_{d}=0.1,1,100$, where the constant contours of the average polymer extension $[\sqrt{\operatorname{trace}(\mathbf{A})}]$ are also plotted to show the evolution of the viscoelastic stresses. This figure qualitatively shows that the viscoelastic stresses increase with both the Weissenberg number and the polymeric viscosity ratio but the dependence on the Weissenberg number is more dramatic. As the viscoelastic stresses increase, the cell deformation decreases resulting in a significant enhancement of the cell viability.

In a typical experiment, the fluid viscoelasticity is usually increased by adding more polymers into the solvent, increasing both the polymeric viscosity and the relaxation time. To mimic this, further simulations are performed to examine the combined effects of the Weissenberg number and the polymeric viscosity ratio of the encapsulating droplet in the range of $0.1 \leq W i_{d} \leq 100$ and $0 \leq \beta_{d}$ $\leq 0.9$, while keeping all other parameters fixed at the baseline values. Note that we reduce the solvent viscosity while increasing the polymeric viscosity such that the total viscosity of the bioink remains fixed. In fact, the polymeric viscosity ratio essentially modifies the effective Weissenberg number defined as $W i^{\prime}=\beta W i{ }^{50}$ As more polymers are added to the bioink fluid, the viscoelastic stresses are expected to get enhanced akin to the high Weissenberg number cases, thus the fluid between the inner droplet and the wall is drained more rapidly pulling the entire inner droplet down nearly uniformly. The deformation and the rate of deformation of the cell are plotted as a function of the dimensionless time in Fig. 5 for $W i_{d}=0.1,1$,
100 and $0.1 \leq \beta_{d} \leq 0.9$. This figure confirms the earlier qualitative observation in Figs. 3 and 4 that the cell deformation decreases as either or both of $\beta_{d}$ and $W i_{d}$ increase. In addition, the rate of the cell deformation also decreases with these parameters but at a significantly smaller rate. The cell deformation looks similar for all the cases in the early time when the viscoelastic stresses are not sufficiently developed but then the effects of viscoelasticity become more pronounced in such a way that increasing $W i_{d}$ and $\beta_{d}$ decreases the cell deformation. The deformation eventually reaches a nearly steady state value for all the cases. Notice that the cell remains significantly deformed even though the deformation parameter seems to have reached a steady state. This is because the surface tension of the cell interface is extremely small (e.g., $\sigma_{o} / \sigma_{i}=2541$ ), so it takes a very long time for the cell to return to its equilibrium spherical cap shape. It is also interesting to observe that, for the all values of $W i_{d}$, the rate of the cell deformation $(\gamma)$ exhibits a damped oscillatory behavior.

Next, we examine and quantify the cell viability using the model proposed by Takamatsu and Rubinsky. ${ }^{26}$ The estimated cell viability is plotted in Fig. 6 as a function of the polymeric viscosity ratio for various Weissenberg numbers in the range $W i_{d}=0$ (Newtonian) and $W i_{d}=100$ (highly viscoelastic). This figure shows that the viscoelasticity generally enhances the cell viability. As the polymeric viscosity ratio increases, the cell viability increases monotonically. However, as the Weissenberg number increases, the cell viability first increases monotonically and then saturates at about $W i_{d}=10$, i.e., the cell viability is not affected significantly when the Weissenberg number is further increased beyond this value.

Different cells exhibit different viscoelastic behavior. We finally investigate the effects of the cell viscoelasticity on the cell deformation and the cell viability. For this purpose, simulations are performed for ranges of the cell Weissenberg number and the cell polymeric viscosity ratio while the other parameters are fixed at the

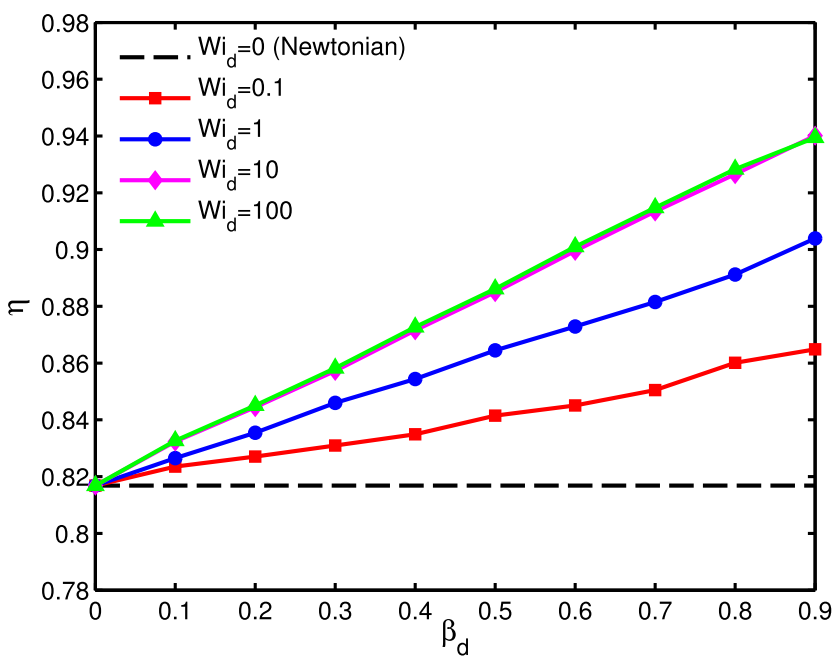

FIG. 6. The estimated cell viability vs the polymeric viscosity ratio in the range $0 \leq \beta_{d} \leq 0.9$ for the Weissenber numbers $W i_{d}=0,0.1,1,10$, and 100 . (We $=2$, $R e=30, L=15, W i_{c}=100, \beta_{c}=0.5, d_{0} / d_{i}=2.85, \sigma_{0} / \sigma_{i}=2541, \mu_{c} / \mu_{d}=5$ $\mu_{d} / \mu_{0}=40, \rho_{c} / \rho_{d}=1, \rho_{d} / \rho_{0}=20, \theta_{e}=60^{\circ}$.) 

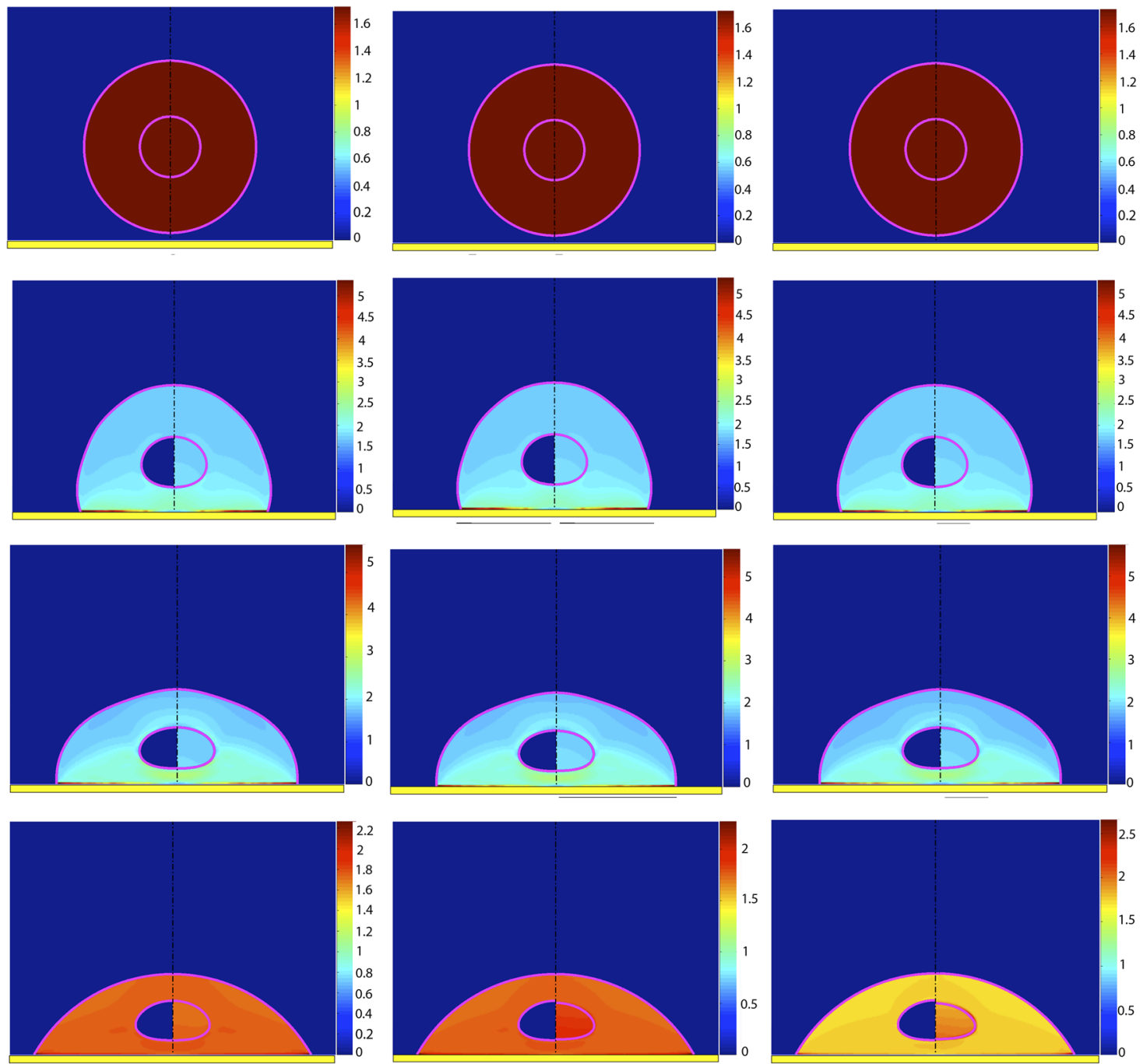

FIG. 7. Effects of viscoelasticity contained in the inner droplet (cell) on evolution of a viscoelastic compound droplet impacting on a flat substrate. The left and right halves of the inner droplet show the Newtonian $\left(W_{i}=0\right)$ and the viscoelastic $\left(W_{i c}=1,10\right.$ and 100 from left to right, respectively, $\left.\beta_{c}=0.5\right)$ cases. The time progresses from top to bottom and the snapshots are taken at times $t^{*}=0,0.32,0.54$ and 3.78. The constant contours denote the average polymer extension $[\sqrt{\operatorname{trace}(\mathbf{A})}]$. The encapsulating droplet is viscoelastic $\left(W e=2, R e=30, W i_{d}=1, L=15, d_{0} / d_{i}=2.85, \sigma_{0} / \sigma_{i}=2541, \mu_{c} / \mu_{d}=5, \mu_{d} / \mu_{0}=40, \rho_{c} / \rho_{d}=1, \rho_{d} / \rho_{0}=20, \theta_{e}=60^{\circ}\right)$.
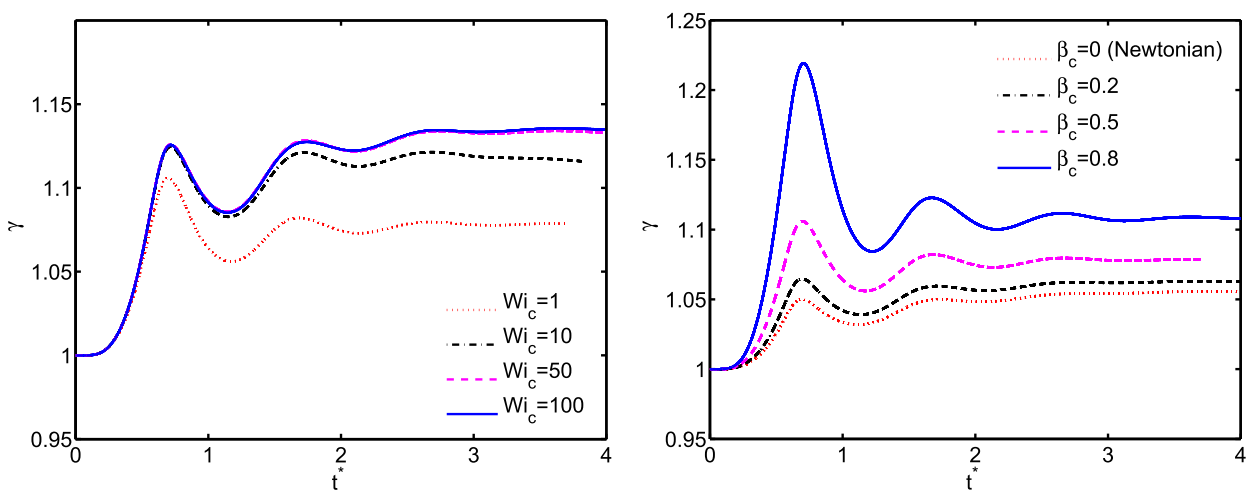

FIG. 8. Effects of cell viscoelasticity on cell deformation. The time evolution of the cell deformation is plotted for the cell Weissenberg numbers $W_{i c}=1,10,50$ and 100 (left) and the cell polymeric viscosity ratios $\beta_{c}=0,0.2,0.5$ and 0.8 (right). The polymeric viscosity ratio is $\beta_{c}=0.5$ in the left plot and the Weissenberg number is $W i_{c}=100$ in the right plot $\left(W e=2, R e=30, W i_{d}=1, \beta_{d}=0.5\right.$, $L=15, d_{0} / d_{i}=2.85, \sigma_{0} / \sigma_{i}=2541, \mu_{c} / \mu_{d}$ $=5, \mu_{d} / \mu_{0}=40, \rho_{c} / \rho_{d}=1, \rho_{d} / \rho_{0}=20$, $\left.\theta_{e}=60^{\circ}\right)$. 


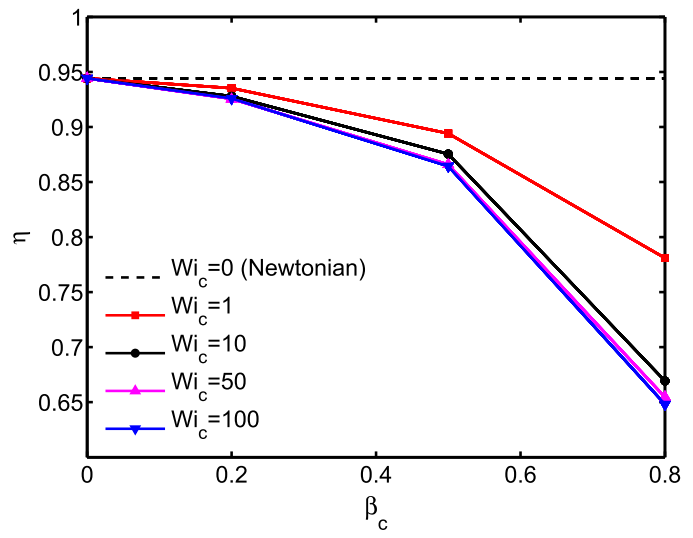

FIG. 9. The estimated cell viability vs the cell polymeric viscosity ratio in the range of $0 \leq \beta_{c} \leq 0.8$ for the cell Weissenberg numbers $W i_{c}=0,1,10,50$ and 100 $\left(W e=2, R e=30, W i_{d}=1, \beta_{d}=0.5, L=15, d_{o} / d_{i}=2.85, \sigma_{o} / \sigma_{i}=2541, \mu_{c} / \mu_{d}=5\right.$, $\left.\mu_{d} / \mu_{0}=40, \rho_{c} / \rho_{d}=1, \rho_{d} / \rho_{0}=20, \theta_{e}=60^{\circ}\right)$.

baseline values. The effects of viscoelasticity contained in the inner droplet (cell) on the evaluation of a viscoelastic compound droplet impacting on a flat substrate are shown in Fig. 7 for $\beta_{c}=0.5$, and $W i_{c}=0$ (Newtonian), 1, 10, and 100. The encapsulating droplet is also viscoelastic with $W i_{d}=1, \beta_{d}=0.5$ and $L=15$. This figure shows that the evolution of the inner droplet as well as the entire compound droplet system is very similar in the early stages for all $W i_{c}$ values but the inner droplet deforms more as $W i_{c}$ increases in the final stage mainly due to a nonuniform viscoelastic stress distribution in the vertical direction in the inner droplet that pulls the upper surface of the inner droplet downward causing more deformation. It is interesting to see that the viscoelasticity contained in the inner droplet enhances/reduces the cell deformation/the cell viability, which is in contrast with the viscoelasticity contained in the encapsulating droplet (bioink) that reduces/enhances the cell deformation/the cell viability. This observation is quantified in Fig. 8. As seen, the cell deformation monotonically increases as either or both of the cell Weissenberg number and the cell polymeric viscosity ratio increase. As $W i_{c}$ increases, the cell deformation increases significantly but eventually saturates at about $W i_{c}=50$. A further increase in $W i_{c}$ beyond this value results in a negligible increase in the cell deformation. On the other hand, the cell deformation monotonically increases with the cell polymeric viscosity ratio. The estimated cell viability is plotted against the cell polymeric viscosity ratio in Fig. 9 for $W i_{c}=0,1,10,50$, and 100 . As seen, the cell viability decreases significantly as the viscoelasticity of the cell increases, suggesting that the Newtonian cell models overpredict the cell viability and should be used cautiously.

\section{CONCLUSIONS}

A compound droplet model is used to examine the effects of viscoelasticity on the cell viability during the impact of a cell-loaded droplet on a flat substrate in droplet-based bioprinting. The viscoelasticity is contained both in the biological cell and the encapsulating droplet but the focus is placed on the effects of the viscoelasticity in the encapsulating droplet fluid. Viscoelasticity is characterized by the Weissenberg number and polymeric viscosity ratio, and comprehensive simulations are performed to investigate their influence on the cell deformation and the deformation-induced cell damage. Takamatsu and Rubinsky's model ${ }^{26}$ is used to quantify the cell viability. Further simulations are performed to show the importance of the cell viscoelasticity in modeling the droplet-based bioprinting. The main findings can be summarized as follows:

1. The viscoelasticity of the encapsulating droplet fluid (bioink) generally enhances the cell viability.

2. The cell viability increases monotonically as polymeric viscosity ratio of bioink increases.

3. The cell viability first increases monotonically as the Weissenberg number increases but then saturates at about $W i_{d}=10$. The cell viability becomes insensitive to a further increase in the Weissenberg number beyond this limit.

4. The viscoelasticity of the cell generally reduces the cell viability. Therefore, the Newtonian cell models may significantly overpredict the cell viability.

In a typical bioprinting experiment, surface coatings such as 3-(Trimethoxysilyl)propyl methacrylate (TMSPMA) are used to covalently link bioprinted constructs upon deposition to glass plates in order to prevent them from lifting off the glass plate and ensure successful long-term culture. In future research, the effects of dynamic contact angle variations due to the interaction between varying polymer amounts in the solvent (i.e., modeled here with increasing polymeric viscosity and relaxation time) and various surface coating materials of the receiving surfaces can be studied.

\section{ACKNOWLEDGMENTS}

We thank the Scientific and Technical Research Council of Turkey (TUBITAK), Grant No. 112M181, and the COST Action Grant No. MP1106 for partial support of this research. The third author (S.T.) acknowledges the Connecticut Innovations Biopipeline Award entitled "A Versatile and Low-Cost Bioprinter for Personalized Medicine” for financial support of this research.

\section{REFERENCES}

${ }^{1}$ T. Boland, T. Xu, B. Damon, and X. Cui, “Application of inkjet printing to tissue engineering," Biotechnol. J. 1, 910-917 (2006).

${ }^{2}$ P. Calvert, "Printing cells," Science 318(5848), 208-209 (2007).

${ }^{3}$ B. Derby, "Printing and prototyping of tissues and scaffolds," Science 338(6109), 921-926 (2012).

${ }^{4}$ S. Tasoglu and U. Demirci, "Bioprinting for stem cell research," Trends Biotechnol. 31(1), 10-19 (2013).

${ }^{5}$ S. V. Murphy and A. Atala, "3D bioprinting of tissues and organs," Nat. Biotechnol. 32(8), 773-785 (2014).

${ }^{6}$ H. Gudapati, M. Dey, and I. Ozbolat, “A comprehensive review on droplet-based bioprinting: Past, present and future," Biomaterials 102, 20-42 (2016).

${ }^{7}$ S. Knowlton, S. Onal, C. H. Yu, J. J. Zhao, and S. Tasoglu, "Bioprinting for cancer research," Trends Biotechnol. 33(9), 504-513 (2015).

${ }^{8}$ S. Knowlton, A. Joshi, B. Yenilmez, I. T. Ozbolat, C. K. Chua, A. Khademhosseini, and S. Tasoglu, "Advancing cancer research using bioprinting for tumor-on-achip platforms,” Int. J. Bioprint. 2(2), 3-8 (2016).

${ }^{9}$ S. Knowlton, S. Anand, T. Shah, and S. Tasoglu, "Bioprinting for neural tissue engineering," Trends Neurosci. 41(1), 31-46 (2018).

${ }^{10} \mathrm{~S}$. Knowlton, B. Yenilmez, and S. Tasoglu, "Towards single-step biofabrication of organs on a chip via 3D printing," Trends Biotechnol. 34(9), 685-688 (2016). 
${ }^{11}$ S. Knowlton and S. Tasoglu, "A bioprinted liver-on-a-chip for drug screening applications," Trends Biotechnol. 34(9), 681-682 (2016).

${ }^{12}$ S. Jana and A. Lerman, "Bioprinting a cardiac valve," Biotechnol. Adv. 33(8), 1503-1521 (2015).

${ }^{13}$ E. Lepowsky, M. Muradoglu, and S. Tasoglu, "Towards preserving post-printing cell viability and improving the resolution: Past, present, and future of $3 \mathrm{D}$ bioprinting theory," Bioprinting 11, e00034 (2018).

${ }^{14}$ M. Nakamura, A. Kobayashi, F. Takagi, A. Watanabe, Y. Hiruma, K. Ohuchi, Y. Iwasaki, M. Horie, I. Morita, and S. Takatani, Biocompatible Inkjet Printing Technique for Designed Seeding of Individual Living Cells (Sage, 2005), Vol. 11, pp. 1658-1666.

${ }^{15}$ B. Derby, "Inkjet printing of functional and structural materials: Fluid property requirements, feature stability, and resolution,” Annu. Rev. Mater. Res. 40, 395414 (2010).

${ }^{16} \mathrm{U}$. Demirci and G. Montesano, "Single cell epitaxy by acoustic picolitre droplets," Lab Chip 7(9), 1139-1145 (2007).

${ }^{17}$ Y. Fang, J. P. Frampton, S. Raghavan, R. Sabahi-Kaviani, G. Luker, C. X. Deng, and S. Takayama, "Rapid generation of multiplexed cell cocultures using acoustic droplet ejection followed by aqueous two-phase exclusion patterning," Tissue Eng., Part C 18(9), 647-657 (2012).

${ }^{18}$ A. Faulkner-Jones, S. Greenhough, J. A. King, J. Gardner, A. Courtney, and W. M. Shu, "Development of a valve-based cell printer for the formation of human embryonic stem cell spheroid aggregates," Biofabrication 5(1), 015013 (2013).

${ }^{19}$ W. Lee, J. C. Debasitis, V. K. Lee, J. H. Lee, K. Fischer, K. Edminster, J. K. Park, and S. S. Yoo, "Multi-layered culture of human skin fibroblasts and keratinocytes through three-dimensional freeform fabrications," Biomaterials 30(8), 1587-1595 (2009).

${ }^{20}$ J. Hendriks, C. W. Visser, S. Henke, J. Leijten, D. B. F. Saris, C. Sun, D. Lohse, and M. Karperien, "Optimizing cell viability in droplet-based cell deposition," Sci. Rep. 5, 11304 (2015).

${ }^{21}$ I. Donderwinkel, J. C. M. van Hestb, and N. R. Cameron, "Bioinks for 3D bioprinting: Recent advances and future prospects," Polym. Chem. 8(8), 4451-4471 (2017).

${ }^{22} \mathrm{H}$. Lu and Z. Peng, "Boundary integral simulations of a red blood cell squeezing through a submicron slit under prescribed inlet and outlet pressures," Phys. Fluids 31(3), 031902 (2019).

${ }^{23} \mathrm{~T}$. Ye and L. Peng, "Motion, deformation, and aggregation of multiple red blood cells in three-dimensional microvessel bifurcations," Phys. Fluids 31(2), 021903 (2019).

${ }^{24}$ W. Wang, Y. Huang, M. Grujicic, and D. B. Chrisey, "Study of impact induced mechanical effects in cell direct writing using smooth particle hydrodynamic method," J. Manuf. Sci. Eng. 130, 021012 (2008).

${ }^{25}$ S. Tasoglu, G. Kaynak, A. J. Szeri, U. Demirci, and M. Muradoglu, "Impact of a compound droplet on a flat surface: A model for single cell epitaxy," Phys. Fluids 22(8), 082103 (2010).

${ }^{26} \mathrm{H}$. Takamatsu and B. Rubinsky, "Viability of deformed cells," Cryobiology 39(3), 243-251 (1999).

${ }^{27} \mathrm{P}$. He, Y. Liu, and R. Qiao, "Fluid dynamics of the droplet impact processes in cell printing," Microfluid. Nanofluid. 18, 569-585 (2016).

${ }^{28}$ C. Zhou, P. Yue, and J. J. Feng, "Deformation of a compound drop through a contraction in a pressure-driven pipe flow," Int. J. Multiphase Flow 34(1), 102-109 (2008).

${ }^{29}$ J. Wang, J. Liu, J. Han, and J. Guan, "Rheology investigation of the globule of multiple emulsions with complex internal structures through a boundary element method," Chem. Eng. Sci. 96, 87-97 (2013).

${ }^{30} \mathrm{Y}$. Chen, X. Liu, and M. Shi, "Hydrodynamics of double emulsion droplet in shear flow," Appl, Phys. Lett. 102(5), 051609 (2013).

${ }^{31}$ Y. Chen, X. Liu, C. Zhang, and Y. Zhao, "Enhancing and suppressing effects of an inner droplet on deformation of a double emulsion droplet under shear," Lab Chip 15(5), 1255-1261 (2015).

${ }^{32}$ Y. Chen, X. Liu, and Y. Zhao, "Deformation dynamics of double emulsion droplet under shear," Appl. Phys. Lett. 106(14), 141601 (2015).

${ }^{33}$ D. Lee, S. N. Beesabathuni, and A. Q. Shen, "Shape-tunable wax microparticle synthesis via microfluidics and droplet impact," Biomicrofluidics 9(6), 064114 (2015).
${ }^{34}$ J. Wang, X. Wang, M. Tai, and J. Guan, "Oriented shift and inverse of the daughter droplet due to the asymmetry of grand-daughter droplets of multiple emulsions in a symmetric flow field," Appl. Phys. Lett. 108(2), 021603 (2016).

${ }^{35}$ A. Rahmat, M. Barigou, and A. Alexiadis, "Deformation and rupture of compound cells under shear: A discrete multiphysics study," Phys. Fluids 31(5), 051903 (2019).

${ }^{36}$ E. M. Toose, B. J. Geurts, and J. G. M. Kuerten, “A 2D boundary element method for simulating the deformation of axisymmetric compound non-Newtonian drops," Int. J. Numer. Methods Fluids 30(6), 653-674 (1999).

${ }^{37}$ C. Zhou, P. Yue, and J. J. Feng, "Simulation of neutrophil deformation and transport in capillaries using Newtonian and viscoelastic drop models," Ann. Biomed. Eng. 35(5), 766-780 (2007).

${ }^{38}$ H. Domejean, M. D. Saint Pierre, A. Funfak, N. Atrux-Tallau, K. Alessandri, P. Nassoy, J. Bibette, and N. Bremond, "Controlled production of sub-millimeter liquid core hydrogel capsules for parallelized 3D cell culture,” Lab Chip 17(1), 110-119 (2017).

${ }^{39}$ D. B. Khismatullin and G. A. Truskey, "Three-dimensional numerical simulation of receptor-mediated leukocyte adhesion to surfaces: Effects of cell deformability and viscoelasticity," Phys. Fluids 17(3), 031505 (2005).

${ }^{40}$ Z. Y. Luo, L. He, S. Q. Wang, S. Tasoglu, F. Xu, U. Demirci, and B. F. Bai, "Twodimensional numerical study of flow dynamics of a nucleated cell tethered under shear flow," Chem. Eng. Sci. 119, 236-244 (2014).

${ }^{41}$ K. Tatsumi, K. Haizumi, K. Sugimoto, and K. Nakabe, "Measurement and analysis of lymphocyte deformation in microchannel contraction flows using a compound drop model," Flow, Turbul. Combust. 96(1), 245-260 (2016).

${ }^{42}$ M. Nooranidoost, M. Haghshenas, M. Muradoglu, and R. Kumar, "Cell encapsulation modes in a flow focusing microchannel: Effects of shell fluid viscosity," Microfluid. Nanofluid. 23(3), 31 (2019).

${ }^{43} \mathrm{M}$. Nooranidoost, D. Izbassarov, and R. Kumar, "Cell encapsulation in a flow focusing microchannel: Effects of viscoelasticity," Bull. Am. Phys. Soc. 63, 271 (2018).

${ }^{44}$ M. D. Chilcott and J. M. Rallison, "Creeping flow of dilute polymer solutions past cylinders and spheres," J. Non-Newtonian Fluid Mech. 29, 381-432 (1988).

${ }^{45}$ P. Carreau and M. Grmela, "Conformation tensor rheological models," in Rheological Modelling: Thermodynamical and Statistical Approaches (Springer, 1991), pp. 126-157.

${ }^{46}$ S. O. Unverdi and G. Tryggvason, "A front-tracking method for viscous, incompressible, multi-fluid flows,” J. Comput. Phys. 100(1), 25-37 (1992).

${ }^{47}$ G. Tryggvason, B. Bunner, A. Esmaeeli, D. Juric, N. Al-Rawahi, W. Tauber, J. Han, S. Nas, and Y.-J. Jan, "A front-tracking method for the computations of multiphase flow,” J. Comput. Phys. 169(2), 708-759 (2001).

${ }^{48}$ D. Izbassarov and M. Muradoglu, "A front-tracking method for computational modeling of viscoelastic two-phase flow systems," J. Non-Newtonian Fluid Mech. 223, 122-140 (2015).

${ }^{49}$ G. Tryggvason, R. Scardovelli, and S. Zaleski, Direct Numerical Simulations of Gas-Liquid Multiphase Flows (Cambridge University Press, Cambridge, UK, 2011).

${ }^{50} \mathrm{D}$. Izbassarov and M. Muradoglu, "A computational study of two-phase viscoelastic systems in a capillary tube with a sudden contraction/expansion," Phys. Fluids 28(1), 012110 (2016).

${ }^{51}$ D. Izbassarov and M. Muradoglu, "Effects of viscoelasticity on drop impact and spreading on a solid surface," Phys. Rev. Fluids 1(2), 023302 (2016).

${ }^{52} \mathrm{M}$. Nooranidoost, D. Izbassarov, and M. Muradoglu, "Droplet formation in a flow focusing configuration: Effects of viscoelasticity," Phys. Fluids 28(12), 123102 (2016).

${ }^{53} \mathrm{H}$. Zolfaghari, D. Izbassarov, and M. Muradoglu, "Simulations of viscoelastic two-phase flows in complex geometries," Comput. Fluid 156, 548-561 (2017).

${ }^{54} \mathrm{X}$. Chen, J. Lu, and G. Tryggvason, "Numerical simulation of self-propelled nonequal sized droplets," Phys. Fluids 31, 052107 (2019).

${ }^{55}$ R. Borges, M. Carmona, B. Costa, and W. S. Don, "An improved weighted essentially non-oscillatory scheme for hyperbolic conservation laws," J. Comput. Phys. 227, 3191-3211 (2008).

${ }^{56}$ R. Fattal and R. Kupferman, "Time-dependent simulation of viscoelastic flows at high Weissenberg number using the log-conformation representation," J. NonNewtonian Fluid Mech. 126, 23-37 (2005). 
${ }^{57} \mathrm{M}$. Muradoglu and S. Tasoglu, "A front-tracking method for computational modeling of impact and spreading of viscous droplets on solid walls," Comput. Fluids 39(4), 615-625 (2010).

${ }^{58}$ S. F. Kistler, "Hydrodynamics of wetting," in Wettability, edited by J. C. Berg (Dekker, New York, 1993), pp. 311-429.

${ }^{59} \mathrm{~N}$. Matubayasi and A. Nishiyama, "Thermodynamic quantities of surface formation of aqueous electrolyte solutions VI. Comparison with typical nonelectrolytes, sucrose and glucose," J. Colloid Interface Sci. 298(2), 910-913 (2006).
${ }^{60} \mathrm{H}$. Lan and D. B. Khismatullin, "A numerical study of the lateral migration and deformation of drops and leukocytes in a rectangular microchannel," Int. J. Multiphase Flow 47, 73-84 (2012).

${ }^{61}$ A. Miri, P. Frommhold, I. Mirzaee, S. Hassan, S. Oskui, D. Nieto, A. Khademhosseini, and Y. Zhang, "Effective bioprinting resolution in tissue model fabrication," Lab Chip 19(11), 2019 (2019).

${ }^{62}$ C. Mandrycky, Z. Wang, K. Kim, and D. Kim, "3D bioprinting for engineering complex tissues," Biotechnol. Adv. 34(4), 422-434 (2016). 